\title{
Overview of Magnetoencephalography_Brief History of its Sensors and Hardware
}

\author{
Koichi Yokosawa *, \#
}

\begin{abstract}
Magnetoencephalography (MEG) has advanced dramatically in the past 50 years, since the first neuromagnetic recording in 1968. Recent MEGs have both high spatial resolution of a few millimeters and high temporal resolution in the order of millisecond. MEG is applied not only clinically, but also in many academic fields including physiology and psychology. In this article, the basic principle and structure of MEG, and the brief history of development are described.
\end{abstract}

Keywords: magnetoencephalography, magnetic sensors, SQUID, OPM.

Adv Biomed Eng. 9: pp. 217-224, 2020.

\section{Introduction}

Neuromagnetic signals were first measured in 1968. Since then, magnetoencephalography (MEG) has been developed, and great advances have been achieved in the last 50 years. In order to measure the weak neuromagnetic signals generated by the brain, which is one hundred millionth of the strength of the earth's magnetic field, highly sensitive magnetic field detection technology and environmental magnetic noise reduction technology are indispensable. With the development of these technologies, data has been accumulated and the usefulness of MEG measurement gradually became evident. With the increase in expectations for novel medical examination devices, rapid progress was achieved in realizing more practical MEG systems. At the same time, the technology for analyzing the recorded MEG signals also advanced. To address the need for cooling of the MEG sensor with liquid helium, MEG systems that can operate with sensors cooled in liquid nitrogen and with sensors at room temperature are being developed, which can reduce maintenance costs. This article overviews the history of development over the last half-century in biomagnetic field measurement, focusing on MEG sensors and hardware. The contents of previous publications $[1,2]$ are partially reproduced with permission.

\section{Outline of MEG}

Brain activity is a weak electric current that flows through neurons. According to the right-handed screw rule, a

Received on July 15, 2019; revised on August 30, 2019; accepted on August 30, 2019.

*Faculty of Health Sciences, Hokkaido University, Sapporo, Japan.

\#E-mail: yokosawa@med.hokudai.ac.jp weak magnetic field is generated around the electric current. By simultaneously measuring this magnetic field at multiple points on the scalp, and back-calculating the signal source from the distribution of the magnetic field, it is possible to know the position of the activated nerves; that is, the active region of the brain. Since this method measures the magnetic field spontaneously generated from the brain, it is completely harmless (non-invasive) and can be repeatedly measured even in healthy subjects.

\subsection{Comparison of characteristics of brain function measuring devices}

In addition to MEG, other devices that allow non-invasive measurement of brain function include electroencephalograph (EEG), functional magnetic resonance imaging device (fMRI), and near-infrared spectroscopy device (NIRS). When classified according to the target of measurement, the EEG measures the electrical signals generated by neural activity in the brain, similar to MEG. On the other hand, fMRI and NIRS detect the dynamics of cerebral blood flow that supports neural activity. While cerebral blood flow dynamics are secondary activities generated via neural activity, electrical current and magnetic field that accompany neural activity can be regarded to be brain activity per se. The EEG and MEG are able to measure fluctuating brain activity in orders of milliseconds. A major characteristic is the ability to track signal processing processes in the brain with high temporal resolution.

In terms of spatial resolution, fMRI and MEG have the advantage of being able to estimate the source of brain activity relatively accurately. In particular, fMRI has the great merit of being able to measure anatomical information at the same time.

On the other hand, when classified according to the size of the device and the degree of freedom of the sub- 
ject, the EEG and NIRS are relatively small, portable, and inexpensive. Since the sensor system is attached to the head, measurement is possible even when the head is moved slightly. On the other hand, fMRI and MEG are large machines installed in a magnetically shielded room, and basically the head should not be moved during fMRI and MEG measurements.

As described above, the four devices for measuring brain function are complementary to each other. The merits and drawbacks of MEG are summarized below. The merits are high spatial resolution (several $\mathrm{mm}$ ) and temporal resolution (1 ms) for estimating brain activity. The drawback are that the device is large and fixed, the subject cannot move the head during measurement, and anatomical information cannot be obtained.

\subsection{Principle and basic configuration}

When neurons in the brain receive action potentials from synapses via transmitters, an ion current is generated inside the cells. At the same time, a volume current flows outside the cells as a return current. The intracellular current can be regarded as a current that flows in an extremely short interval equivalently (current dipole). Both the current dipole and the volume current generate a magnetic field according to the Biot-Savart law. However, since the volume current spreads in the skull and the magnetic fields cancel out each other, the brain magnetic field component in the normal direction of the head surface is mainly derived from the intracellular current. The EEG measures the electric potential on the head surface, which is produced by the volume current that spreads inside the skull. On the other hand, the MEG measures the magnetic field immediately over the head surface, which is generated by the intracellular current. Since the volume current is strongly influenced by the shape of the skull as well as the distribution of electrical conductivity in brain tissues, and becomes distorted, it is difficult to back-calculate the active region of the brain from the EEG. In contrast, because the intracellular current is confined to a short interval and the magnetic permeability of living tissues is almost constant, the magnetic field generated is not distorted when reaching the head surface. Therefore, by arranging a large number of magnetic sensors around the head and measuring the magnetic field distribution, the position of the current dipole; that is, the active region in the brain, can be estimated.

Other advantages of MEG are that multi-point measurements can be performed easily because attachment of electrodes to the subject is not required, and that there is no interference between the sensors and the subject since the two are separated. On the other hand, an electrical interface clearly exists because of the difference in electrical conductivity between the subject and the exter-

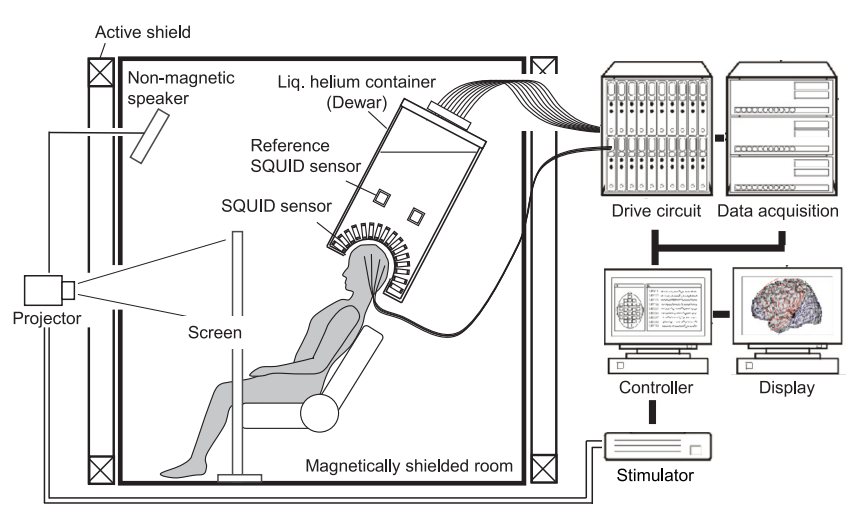

Fig. 1 Typical setup of MEG. High efficiency magnetic shielding and supersensitive magnetic sensors are required. Further details are described in the text.

nal environment (air); whereas a clear magnetic interface does not exist, because there is no difference in magnetic permeability between the subject and the external environment. Therefore, the drawback of MEG is that it is markedly affected by magnetic noise in the external environmental (environmental magnetic noise). Therefore, measurement of magnetic field from the brain requires technology that maximally reduces environmental magnetic noise, in addition to highly sensitive magnetic sensors that can measure a weak magnetic field.

The sensor component of a MEG is composed of a large array of highly sensitive magnetic sensors for measuring the magnetic field distribution, and is installed in a magnetic shield room where the subject is also present. The sensor unit in addition to peripheral devices including a data recording system constitute the basic configuration of the MEG system (Fig. 1). The sensor and the system configuration will be overviewed below. For details, refer to reference books $[3,4]$.

\subsection{Sensors}

The strength of the magnetic field of the brain is only about $10^{-7}$ to $10^{-8}$ times that of the earth's magnetic field. The superconducting quantum interference device (SQUID) was the only device with the sensitivity to measure the magnetic field of the brain, before the advent of the optically pumped magnetic sensor (to be described later) with dramatically improved sensitivity. The MEG sensor is composed of the SQUID connected to a superconducting detection coil and a drive circuit.

The detection coil often has a gradiometer configuration in order to reduce the environmental magnetic noise to $1 / 100$ to $1 / 1000$. The structure consists of a combination of multiple coils that have sensitivity to magnetic fields in opposite direction to the gradiometer, and are sensitive only to adjacent magnetic field sources. If the gradiometer is placed near the brain to be mea- 
sured, the gradiometer has no sensitivity to the environmental magnetic noise that has a magnetic source far away, thereby allowing selective measurement of the magnetic field of the brain.

Since the SQUID and detection coil (this pair will be referred to as "SQUID sensor" hereinafter) are both superconductors, they operate in liquid helium. Therefore, the SQUID sensor is placed in a low temperature container (Dewar) made of fiber-reinforced plastic (FRP) that allows passage of the magnetic field. There is a helmet-shaped dent at the bottom of the Dewar, around which 100 to 300 SQUID sensors are aligned in a helmet shape. In addition, a reference sensor for measuring environmental magnetic noise may be attached separately. The inside of the Dewar is always filled with liquid helium.

Since the sensitivity of the SQUID sensor is defined by the resolution of the magnetic field (to be exact, magnetic flux density), it is expressed as the equivalent magnetic field noise. If the magnetic shielding is sufficient, the equivalent noise is the intrinsic noise of the SQUID and/or the drive circuit. Since the intrinsic noise can be approximated by thermal noise, the unit becomes $\mathrm{T} / \sqrt{ } \mathrm{Hz}$. In the case of a gradiometer that measures magnetic field gradient, the unit $\mathrm{fT} / \mathrm{cm} \sqrt{\mathrm{Hz}}$ is used, where $\mathrm{f}$ (femto) is $10^{-15}$. The sensitivity of the SQUID sensor used in MEG is in the white noise range (frequency range of several tens of $\mathrm{Hz}$ or higher), and approximately 3 to $10 \mathrm{fT} / \sqrt{\mathrm{Hz}}$.

\subsection{Magnetic shield and peripheral devices}

The size of the magnetic shield room is about 2 to $3 \mathrm{cu}-$ bic meter and is a metal cubic or rectangular cuboid box with a door. The box houses the sensor system and the subject. The wall consists of a layer of soft magnetic material with high magnetic permeability (permalloy) for shielding the magnetic field, and a layer of metal such as aluminum and copper with high conductivity for shielding the electromagnetic wave (electromagnetic shield). The wall often has two to three layers of 2-3 mm thick permalloy material and 1 layer of electromagnetic shield around $5 \mathrm{~mm}$ in thickness. Increasing the thickness of the electromagnetic shield layer improves the shielding performance of alternating magnetic field produced by the eddy current effect. The magnetic shielding performance depends on the number of layers of permalloy and the size of the shield room, and is approximately $1 / 100$ to $1 / 10000$. In order to improve the shielding performance, an active shield that monitors the environmental magnetic noise and nullifies the noise using feedback mechanism may be added.

Peripheral devices of the MEG system include a drive circuit of the SQUID sensor, a control system, a data acquisition system, a display system for signal processing and analysis, a chair or bed for positioning the subject or patient, a coordinate system (digitizer) to measure the positional relationship between the sensor system and the head, and an EEG. In addition, for localization of sensory functions in the brain and measurement of higher brain functions, it is necessary to administer stimulation (audiovisual stimulation, somatosensory stimulation, characters, language, etc.) to the subject. Stimulus delivery device and its control system are also included in the system.

\section{History of development of MEG}

Electrocardiograms and EEG, which measure the electrophysiological activities of the human body as body surface potentials, were first measured by Eintoven (1903) and Berger (1929), but measurement of the magnetic field was realized some sixties years later. As mentioned above, the strength of the magnetic field signal of the brain is very weak; signals of brain rhythms such as alpha waves are approximately $10^{-7}$ times that of the earth's magnetic field $(50 \mu \mathrm{T})$, and signals of transient responses such as auditory evoked responses are approximately $10^{-8}$ times. On the other hand, the magnetic field signals of the heart has a strength 10 to 100 times that of the brain, and are relatively easy to measure. Initially, magnetic field was measured using an induction coil which was a copper wire wound millions of times around a material with high magnetic permeability (magnetic field of the heart was reported by Baule \& McFee in 1963 [5], and magnetic field of the brain by Cohen in 1968 [6]). In 1965, Silver \& Zimmerman developed a practical SQUID [7], which was applied to MEG by Cohen in 1972 [8].

As mentioned above, until now SQUID sensor has been almost the only sensor with sensitivity that can measure the weak magnetic field of the brain. Therefore, it can be said that the history of MEG development is the history of the development of SQUID sensors for multi-channel systems specialized for use in MEG. Therefore, first of all, the history of development of commercial MEG device using SQUID sensors will be overviewed. The history of MEG development has been described in detail in references [9-11].

Figure 2 shows the relationship between the year of development (publication) of MEG until 2007 and the number of channels (number of installed SQUID sensors) plotted on a semi-logarithmic graph. The graph shows that the number of channels increases linearly over time, similar to Moore's Law which is known for semiconductors. In the 1970 s, S.H.E. Corp., the predecessor of 4D-Neuroimaging, in the United States marketed the 1-channel SQUID sensor (gradiometer). However, 
(A)

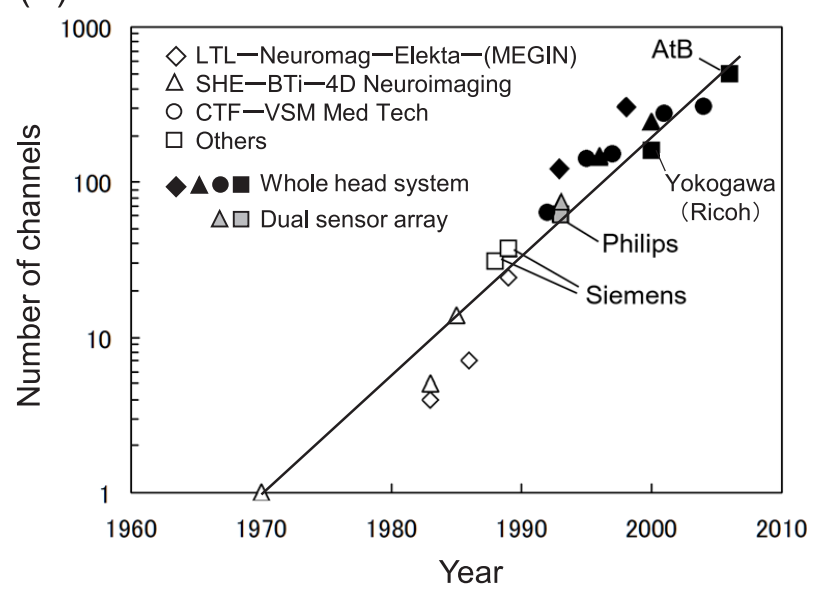

(B)

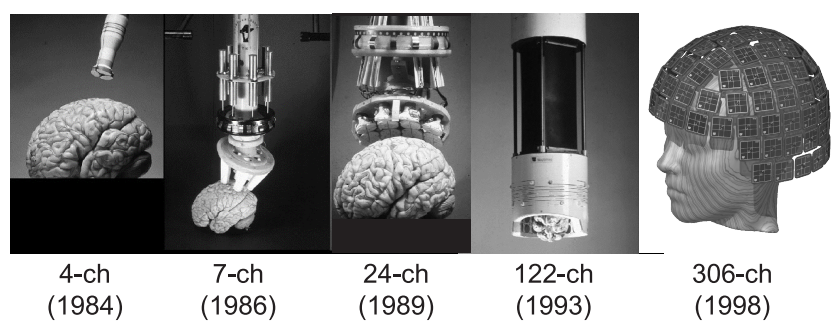

(C)
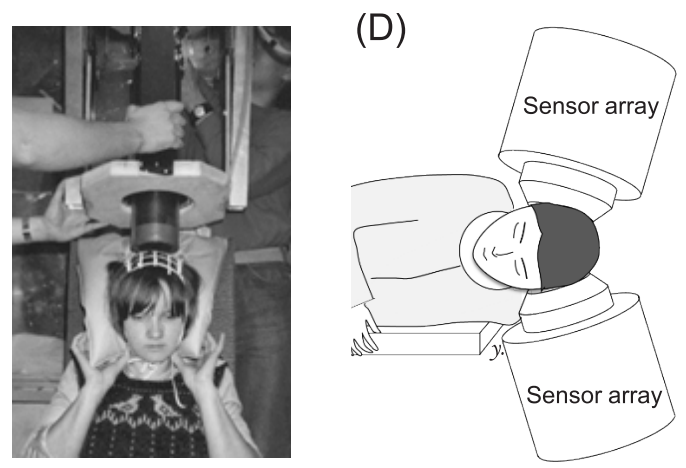

Fig. 2 (A) History of increase in sensor channels installed in MEG. The number of channels are plotted exponentially against year. (B) Examples of multi-channel devices. Implementers: Low Temperature Laboratory (LTL), Helsinki University of Technology $\rightarrow \mathrm{Neu}-$ romag Oy $\rightarrow$ Elekta Neuromag. (C) MEG recording using a single-channel MEG in 1981. (D) A schematic diagram of MEG with dual sensor arrays. Figures B and C: courtesy of Prof. Riitta Hari, Aalto University, Finland.

to estimate the signal source in the brain, it was necessary to measure the brain magnetic field at multiple points to obtain the magnetic field distribution. In that era, the same measurement was repeated while changing the point of measurement. In the 1980s, research on neuromagnetic field became active. When the usefulness in medical and physiological fields began to be recognized, the demand for simultaneous multi-point measurement increased, and SQUID sensors with multiple channels were developed and marketed [12-14]. In addition, the leading manufacturers such as Siemens [15] in Germany and Philips [16] in the Netherlands also joined in the development, and competitions among several manufacturers resulted in the dramatic increase in number of SQUID sensors from several channels to tens of channels. Philips and 4D-Neuroimaging (Biomagnetic Technologies; BTi, at that time) proposed a dual sensor array that doubled the measurement area by arranging two large Dewars on two sides of the head or the chest (Fig. 2D). Nevertheless, until the end of the 1980s, MEG and magnetocardiography were not differentiated, and the configuration was a large number of SQUID sensors arranged at the bottom of the Dewar.

The whole head MEG system, in which the SQUID sensors are arranged in a helmet shape to cover the entire head, was first developed by CTF Systems, Inc. of Canada [17]. For the whole head type, the design of the helmet on which the SQUID sensors are placed is unavoidably rather big. In the case of a subject with a small head, such as a child, the distance between the head and the detection coil becomes greater, and the signals are attenuated, which is a drawback of this design. However, since the whole head can be measured in one operation, subsequent MEGs are designed as the whole head type, except for special-purpose devices.

In the latter half of the 1990s, the number of channels of whole head MEG increased further [18-21], and vector type MEG, which measures multiple magnetic field direction components at one point of measurement, was also proposed [22]. However, the SQUID sensor measures the magnetic flux or its spatial difference, and the signal volume is proportional to the area of the detection coil. Hence, there is a limit to the number of sensors that can be placed on the surface of the head. For this reason, the number of MEG channels using SQUID sensors was saturated at about 300 to 400 . In other words, the number of channels is no longer the benchmark for MEG development at around 2007. Since then, reorganization has proceeded in manufacturers of MEG. The MEG division of Yokogawa Electric Corporation was taken over by Ricoh Co. Ltd. [23], and York Instruments has acquired the Neuromag business from Elekta to this day [24] (Fig. 3). During this period, great advances have been made in brain function analysis technologies such as signal source estimation technology, correlation analysis of brain activity, and network analysis (for details, see reference books $[3,4])$. On the other hand, the circulation type liquid helium recondensation device has become an accessary device for MEG, and new hardware has been proposed, including dedicated devices for special purposes such as pediatric MEG [25] and magneto- 

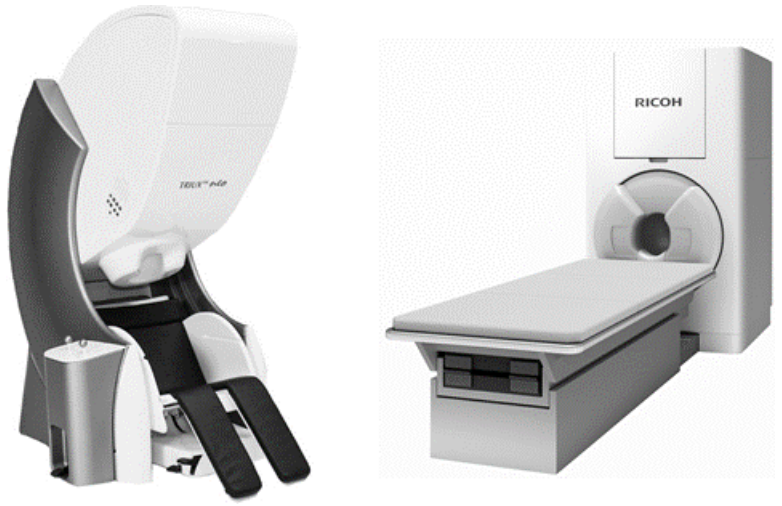

Fig. 3 Examples of current commercial MEGs. Left: TRIUX neo, MEGIN Oy, (reproduced with permission of the manufacturer), Right: RICOH 160-1, Ricoh Co. Ltd., (reproduced by courtesy of the manufacturer).

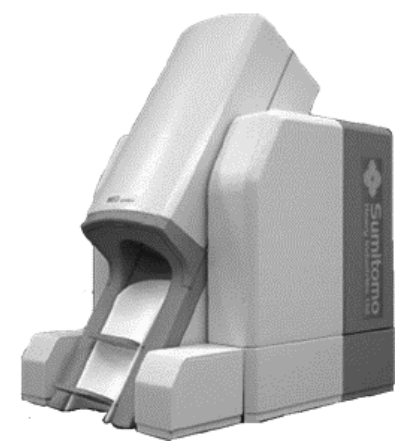

Fig. 4 Superconducting self-shielded MEG (photograph provided by Sumitomo Heavy Industries, Ltd.).

spinograph [26], as well as portable MEG using a cylindrical superconducting magnetic shield [27, 28] (Fig. 4). Dual MEG by connecting two existing MEG units [2931] has also been tried (Fig. 5).

A total of more than 150 MEG units, mainly commercial devices, have been installed around the world (Fig. 6 shows the total number in 2014, but includes estimates). Coverage of MEG examination by health insurance is essential for continuous clinical use. In the United States, three current procedural terminology (CPT) codes were assigned to MEG for focus estimation and functional domain estimation of epilepsy in 2001. Furthermore, ambulatory payment classification (APC) was established and MEG examination became covered by medical insurance in 2003. On the other hand, in Japan, the Ministry of Health and Welfare approved marketing of MEG as a clinical device in 1997, and medical fee points (5,000 points for neuromagnetic diagnosis) were assigned and MEG examination was covered by health insurance in 2004. In addition, the 2015 revision expanded its application to include "differential diagnosis of pri-

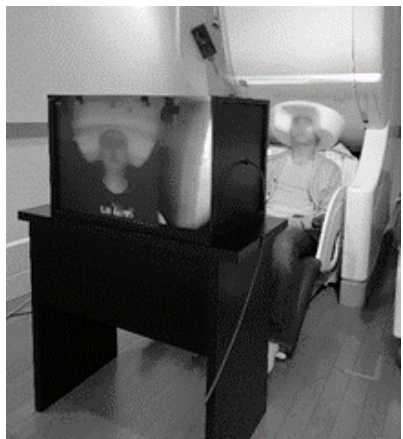

Fig. 5 An example of Dual MEG. Two MEGs are connected directly with fiber optic cables, enabling hyperscanning during virtual face-to-face communication between two subjects.

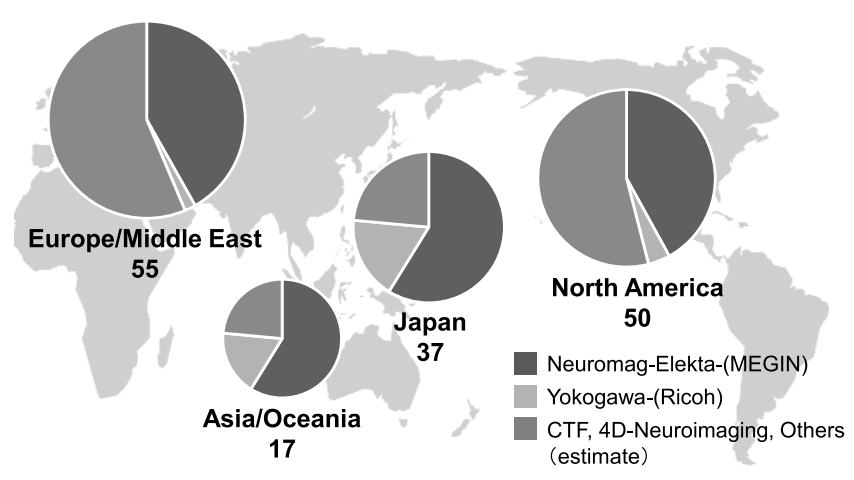

Fig. 6 Number of MEGs installed in the world in 2014 (estimated).

mary and secondary epilepsies, sensory and motor disorders associated with central nervous system disease, or decision of treatment policy including diagnosis of surgical site and selection of surgical methods for patients with epilepsy" (D236-3 Magnetoencephalogram), and 5100 points were assigned.

\section{MEG: present and future}

An oxide superconductor with a superconducting transition temperature even higher than that of liquid nitrogen (77 K) was discovered in 1986, and an oxide-based SQUID sensor using the highly practical yttrium-based superconductor (YBCO) was developed [32, 33] and applied to the measurement of the cardiac magnetic field, which has a signal strength 10 to 100 times higher than the brain magnetic field [34-37]. In addition, magnetoresistance (MR) sensors and magneto-impedance (MI) sensors can also be used to acquire magnetocardiograms with higher sensitivity [38]. Due to the price increase of helium in recent years, there is increased expectation for MEG that does not require helium, and the development of MEG using oxide-based SQUID sensors is still ongo- 
ing [39]. On the other hand, report has shown that an optically pumped magnetic sensor made of alkali metal can achieve a level of sensitivity equal to or higher than that of a conventional (metal based) SQUID sensor, and the development of MEG using this sensor is in progress.

\subsection{Outline of optically pumped magnetic sensor}

The main component of the optically pumped magnetic sensor is a glass cell filled with alkali metal gas. When a magnetic field is applied to the cell to cause Zeeman splitting for the ground level of the alkali metal, and then circularly polarized light is applied to induce the atoms to precess, the amplitude of the circularly polarized light is modulated depending on the magnitude of the magnetic field. In this manner, by inducing spin polarization (deviation of the number of electrons occupying different energy levels) using circularly polarized light (this is called optical pumping), a weak magnetic field can be read from the interaction between the magnetic field and spin polarization, which is the basic principle of optically pumped magnetic sensor. For this reason, it is sometimes called an alkali metal magnetic flux meter or an atomic magnetic flux meter. Several types of optically pumped magnetic sensors are known, such as uni-axial type and orthogonal type. All of them use a circularly polarized laser, which makes the readout circuit a little complicated, but the major feature is that cooling is not required. Since there is no Dewar, in principle the magnetic sensor (cell) and the head can be brought into close proximity to each other. In addition, if the sensor is small, it can be mounted on the head similar to EEG or NIRS, thus allowing movement of the head during measurement (Fig. 7).

The principle of optical pumping was proposed by Kastler in 1950, and was applied to magnetic sensors in 1957. In the area of biomagnetic field measurement, much interest was generated in 2003, when a group at Fribourg University in Switzerland succeeded to use a laser-pumped magnetometer in measuring the cardiac magnetic field [40, 41], and a group at the University of Princeton in the United States reported an optically pumped magnetometer with sensitivity exceeding that of SQUID sensor [42, 43]. At this time, the sensor developed at Princeton University employed potassium and consisted of two optically pumped magnetic sensors in a gradiometer configuration, which achieved a sensitivity of $7 \mathrm{fT} / \sqrt{ }$ Hz. Based on this technology, QuSpin in the United States has pushed ahead with miniaturization of sensors, and currently supplies magnetic sensors with a size of $12.4 \times 16.6 \times 24.4 \mathrm{~mm}$, weight of $4 \mathrm{~g}$, and sensitivity of 10 to $15 \mathrm{fT} / \sqrt{\mathrm{Hz}}$ [44].

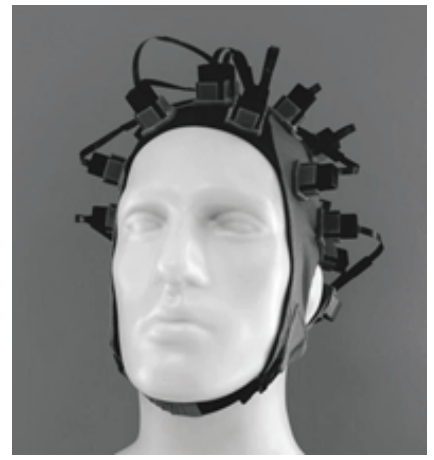

Fig. 7 Optically pumped magnetometers mounted on a head (reproduced with permission from QuSpin Inc.). The subject is free to move the head if the surrounding magnetic field is strictly uniform.

\subsection{MEG without cooling}

Many articles on MEG using optically pumped magnetic sensors have already been published [45-53], but the most well known system was reported by Boto et al. [49] in 2018, which is a "MEG allowing head movement". The system comprises 13 optically pumped magnetic sensors (QuSpin) with sensitivity of $15 \mathrm{fT} / \sqrt{\mathrm{Hz}}$ which are mounted on the scalp surface, and a magnetic shield made of material with high magnetic permeability combined with a 3-axis multi-layered active shield, which creates a uniform magnetic field space (within this space, the error of uniformity of the field is within 5\%) of $40 \mathrm{~cm}^{3}$ around the head of the subject wearing the sensors. Study has shown that as long as measurement is made within this space, this system can measure the same brain magnetic field as a conventional MEG using SQUID sensors (CTF 275 channel) even when the measurement is performed while the head is moving. Due to the large size of the magnetic shield, the system cannot be described as portable, but it represents a new generation of MEG that harnesses the advantages of the optically pumped magnetic sensor that does not require cooling and can be mounted on the head.

\section{Conclusion}

Attempt to measure the biomagnetic field was started in 1963. This article overviews the hardware of MEG and the history of development in the last fifty years. For around 25 years (1983-2008) during this period, the number of channels, an easy-to-understand index, increased exponentially, according to Moore's Law. In Japan, this period coincided with the era of bubble economy, and it was an era that favored especially hardware development. Thereafter, the number of channels reached a plateau, and at first glance there seemed to be a long period in which no major changes were seen in the hardware. In recent years, there have been new developments 
in measurement technologies including sensor technology, which is expected to eliminate the limitations due to measurements and uncover new applications. Developments over the next 50 years are anticipated.

\section{Conflicts of interest}

The authors declare no conflicts of interest with any companies or commercial organizations per the definition of Japanese Society for Medical and Biological Engineering.

\section{References}

1. Yokosawa K, Kuriki S: Magnetoencephalography systems. In: Ogawa S, Ueno S supervising eds. Noninvasive and Visualizing Technologies, NTS, Tokyo, pp. 418-427, 2007 (in Japanese).

2. Yokosawa K: Brief History of Magnetoencephalography. Trans Jpn Soc Med Biol Eng. 57(4-5), 113-118, 2019 (in Japanese).

3. Hansen PC, Kringelbach ML, Salmelin R, Eds.: MEG: An introduction to Methods, Oxford University Press, New York, 2010.

4. Hari R, Puce A: MEG-EEG Primer, Oxford University Press, New York, 2017.

5. Baule G, McFee R: Detection of the magnetic field of the heart. Am Heart J. 66, 95-96, 1962.

6. Cohen D: Magnetoencephalography: evidence of magnetic field produced by alpha-rhythm currents. Science. 161(3843), 784786, 1968.

7. Silver AH, Zimmerman JE: Quantum transitions and loss multiply connected superconductors. Phys Rev Lett. 15(23), 888-891, 1965.

8. Cohen D: Magnetoencephalography: detection of the brain's electrical activity with a superconducting magnetometer. Science. 175(4022), 664-666, 1972.

9. Hari R, Salmelin R: Magnetoenceohalography: from SQUIDS to neuroscience neuroimage 20th anniversary special edition. NeuroImage. 61, 386-396, 2012.

10. Clarke J, Braginski AI, Eds.: The SQUID handbook, Vol. II Applications of SQUIDs and SQUID systems, pp. 269-389, Wiley-VCH Verlag GmbH \& Co. KGaA, Weinheim, 2006.

11. Nowak H: Biomagnetic instrumentations. In: Andrä W, Nowak $\mathrm{H}$, eds. Magnetism in Medicine, A handbook, Second, Completely revised and enlarged edition, pp. 101-163, Wiley-VCH Verlag GmbH \& Co. KGaA, Weinheim, 2007.

12. Ilmoniemi R, Hari R, Reinikainen K: A four-channel squid magnetometer for brain research. Electroencephalogr Clin Neurophysiol. 58, 467-773, 1984.

13. Knuutila J, Ahlfors S, Ahonen A, Hällström J, Kajola M, Lounasmaa OV, Vilkman V: Large-area low-noise seven-channel dc SQUID magnetometer for brain research. Rev Sci Instrum. 58(11), 2145-2156, 1984.

14. Ahlfors S, Ahonen A, Ehnholm G, Hämäläinen M, Ilmoniemi R, Kajola M, Kiviranta M, Knuutila J, Lounasmaa O, Simola J, Tesche C, Vilkman V: A 24-SQUID gradiometer for magnetoencephalography. Physica B. 165\&166, 97-98, 1990.

15. Hoenig HE, Daalmans GM, Bar L, Bommel FR, Paulus A, Uhl D, Weisse HJ, Schneider S, Seifert H, Reichenberger H, Abraham-Fuchs K: Multi-channel DC SQUID sensor array for biomagnetic applications. IEEE Trans Magnetics. 27(2), 2777-
2785, 1991.

16. Dössel O, David B, Fuchs M, Kruger J, Ludeke K-M, Wischmann H-A: A modular 31-channel SQUID system for biomagnetic measurements. IEEE Trans Appl Supercond. 3(1), 1883-1886, 1993.

17. Vrba J, Taylor B, Cheung T, Fife AA, Haid G, Kubik PR, Lee S, McCubbin J, Burbank MB: Noise cancellation by a whole-cortex SQUID MEG system. IEEE Trans Supercond. 5(2), 2118-2123, 1993.

18. Ahonen AI, Hämäläinen MS, Kajola MJ, Knuutila JET, Laine PP, Lounasmaa OV, Parkkonen LT, Simola JT, Tesche CD: 122channel squid instrument for investigating the magnetic signals from the human brain. Phys Scr. 198, T49A, 1993.

19. Knuutila JET, Ahonen AI, Hämäläinen MS, Kajola MJ, Laine PP, Lounasmaa OV, Parkkonen LT, Simola JTA, Tesche CD: A 122-channel whole-cortex SQUID system for measuring the brain's magnetic fields. IEEE Trans Magnetics. 29(6), 33153320, 1993.

20. Vrba J, Taylor B, Cheung T, Fife AA, Haid G, Kubik PR, Lee S, McCubbin J, Burbank MB: Noise cancellation by a whole-cortex SQUID MEG system. IEEE Trans Supercond. 5(2), 2118-2123, 1995.

21. Fife AA, Vrba J, Robinson SE, Haid G, Hoang T, Kishi D, Kubik PR, Lee S, Loewen R, McCubbin J, MacKay J, McKenzie D, Spear P, Taylor B, Tillotson M, Coppola R: A 275 channel whole-cortex MEG system. In: Nowak H, Haueisen J, Gie $\beta$ ler F, Houonker R eds. Proceedings of the 13th International Conference on Biomegnetism, pp. 912-914, VDE Verlag GmbH, Berlin, Offenbach, 2002.

22. Pasquarelli A, Rossi R, Melis MD, Marzrtti L, Trebeschi A, Erne SN, Argos 500: operating of a helmet vector-MEG. In: Halgren R ed. Proceedings of the 14th International Conference on Biomegnetism, pp. 34-35, Biomag 2004 Ltd, Boston, 2004.

23. <https://www.ricoh.co.jp/meg/> [accessed July 11, 2019].

24. <https://megin.fi/triux-neo/> [accessed July 11, 2019].

25. Okada Y, Hämäläinen M, Pratt K, Mascarenas A, Miller P, Han M, Robles J, Cavallini A, Power B, Sieng K, Sun L, Lew S, Doshi C, Ahtam B, Dinh C, Esch L, Grant E, Nummenmaa A, Paulson D: Baby MEG: A whole-head pediatric magnetoencephalography system for human brain development research. Rev Sci Instrum. 87(9), 094301, 2016.

26. Sumiya S, Kawabata S, Hoshino Y, Adachi Y, Sekihara K, Tomizawa S, Tomori M, Ishii S, Sa-kaki K, Ukegawa D, Ushio S, Watanabe T, Okawa A: Magnetospinography visualizes electrophysiological activity in the cervical spinal cord. Sci Rep. 7, 2192, 2017.

27. Ohta H, Matsui T: Whole-head MEG system in a superconducting magnetic shield. Physica C, 341-348, 2713-2714, 2000.

28. Ohta H, Matsui T, Uchikawa Y: A whole-head SQUID system in a superconducting magnetic shield. IEEE Trans Supercond. 17(2), 730-733, 2007.

29. Hirata M, Ikeda T, Kikuchi M, Kimura T, Hiraishi H, Yoshimura Y, Asada M: Hyperscanning MEG for understanding mother-child cerebral interactions. Front Hum Neurosci. 8, 118, 2014.

30. Zhdanov A, Nurminen J, Baess P, Hirvenkari L, Jousmäki V, Mäkelä JP, Mandel A, Meronen L, Hari R, Parkkonen L: Internet-based real-time audiovisual link for dual MEG recordings. Plos One. 10(6), e0128485, 2015. 
31. Takano K, Watanabe H, Yagyu K, Shimojo A, Boasen J, Murakami Y, Shiraishi H, Yokosawa K, Saito T: Semi-automated brain responses in communication: A magnetoencephalographic hyperscanning study. Conf Proc IEEE Eng Med Biol Soc. 28932896, 2020.

32. Drung D, Ludwig F, Müller W, Steinhoff U, Trahms L, Shen YQ, Jensen MB, Vase P, Holst T, Freltoft T, Curio G: Integrated $\mathrm{YBa}_{2} \mathrm{Cu}_{3} \mathrm{O}_{7-\mathrm{x}}$ magnetometer for biomagnetic measurements. Appl Phys Lett. 68(10), 1421-1423, 1996.

33. Yokosawa K, Tsukamoto A, Miyashita T, Kandori A, Suzuki D, Tsukada K, Takagi K: A signal-to-noise chart for designing planar high-Tc SQUID gradiometers for magnetocardiographs. Supercond Sci Technol. 15(1), 136-140, 2001.

34. Yokosawa K, Suzuki D, Tsukamoto A, Miyashita T, Kandori A, Tsukada K, Takagi K: Compact and easy-operation magnetocardiograph with four-channel planar gradiometers. Conf Proc IEEE Eng Med Biol Soc. 4, 3281-3284, 2001.

35. Yokosawa K, Kandori A, Miyashita T, Suzuki D, Tsukada K, Tsukamoto A: A stabilizer for superconducting quantum interference device gradiometers for operation in lightly shielded environments. Appl Phys Lett. 82(18), 3059-3061, 2003.

36. Yokosawa K, Kandori A, Miyashita T, Suzuki D, Tsukada K, Tsukamoto A: Magnetocardiograph based on a $4 \times 4$ array of high-temperature superconducting quantum interference devices. Appl Phys Lett. 82(26), 4833-4835, 2003.

37. Yokosawa K, Tsukamoto A, Suzuki D, Kandori A, Miyashita T, Ogata K, Seki Y, Tsukada K: A 16-channel high-Tc SQUIDmagnetometer system for magnetocardiogram mapping. Supercond Sci Technol. 16(12), 1383-1386, 2003.

38. Shirai Y, Hirao K, Shibuya T, Okawa S, Hasegawa Y, Adachi Y, Sekihara K, Kawabata S: Magnetocardiography using a magnetoresistive sensor array. Int Heart J. 60(1), 50-54, 2019.

39. Dammers J, Chocholacs H, Eich E, Boers F, Faley M, DuninBorkowski RE, Jon Shah N: Source localization of brain activity using helium-free interferometer. Appl Phys Lett. 104, 213705, 2014.

40. Bison G, Wynands R, Weis A: A laser-pumped magnetometer for the mapping of human cardio-graphic fields. Appl Phys B. 76(3), 325-328, 2003.

41. Bison G, Pasquarelli A, Weis A, Erne SN: SQUID vs. optically pumped magnetometer: a comparison of system performance. In: Halgren R ed. Proceedings of the 14th International Conference on Biomegnetism, pp. 31-32, Biomag 2004 Ltd, Boston, 2004.

42. Kominis IK, Kornack TW, Allred JC, Romalis MV: A subfemtotesla multichannel atomic magnetometer. Nature. 422, 596599,2003

43. Romalis MV, Ben-Amar Baranga A, Hoffman DE: Magnetoencephalography with an atomic magnetometer. In: Halgren R ed. Proceedings of the 14th International Conference on Biomegnetism, p. 33, Biomag 2004 Ltd., Boston, 2004.

44. <https://quspin.com/experimental-meg-cap/> [accessed July 11, 2019].

45. Xia H, Baranga B-A, Hoffman D, Romalis MV: Magnetoencephalography with an atomic magnetometer. Appl Phys Lett. 89, 211104, 2006.

46. Shah VK, Wakai RT: A compact, high performance atomic magnetometer for biomedical applications. Phys Med Biol. 58, 8153$8161,2013$.
47. Boto E, Meyer SS, Shah V, Alem O, Knappe S, Kruger P, Fromhold TM, Lim M, Glover PM, Morris PG, Bowtell R, Barnes GR, Brookes MJ: A new generation of magnetoencephalography: room temperature measurements using optically-pumped magnetometers. NeuroImage. 149, 404-414, 2017.

48. Borna A, Carter TR, Goldberg JD, Colombo AP, Jau Y-Y, Berry C, McKay J, Stephen J, Weisend M, Schwindt PDD: A 20-channel magnetoencephalography system based on optically pumped magnetometers. Phys Med Biol. 62, 8909-8923, 2017.

49. Boto E, Holmes N, Leggett J, Roberts G, Shah V, Meyer SS, Duque Muñoz L, Mullinger KJ, Tierney TM, Bestmann S, Barnes GR, Bowtell R, Brookes MJ: Moving magnetoencephalography towards real-world applications with a wearable system. Nature. 555, 657-661, 2018.

50. Labyt E, Corsi M-C, Fourcault W, Laloy AP, Bertrand F, Lenouvel F, Cauffet G, Le Prado M, Berger F, Morales S: Magnetoencephalography with optically pumped ${ }^{4} \mathrm{He}$ magnetometers at ambient temperature. IEEE Trans Med Imaging. 38(1), 90-98, 2019.

51. Tierney TM, Holmes N, Mellor S, López JD, Roberts G, Hill RM, Boto E, Leggett J, Shah V, Brookes MJ, Bowtell R, Barnes GR: Optically pumped magnetometers: from quantum origins to multi-channel magnetoencephalography. NeuroImage. 149, 598608, 2019.

52. Barry, DN, Tierney TM, Holmes N, Boto E, Roberts G, Leggett J, Bowtell R, Brookes MJ, Barnes GR, Maguire EA: Imaging the human hippocampus with optically-pumped magnetoencephalography. NeuroImage. 203, 116192, 2019.

53. Borna A, Carter TR, Colombo AP, Jau Y-Y, McKay J, Weisend M, Taulu S, Stephen JM, Schwindt PDD: Non-invasive functional-brain-imaging with an OPM-based magnetoencephalography system. Plos One. 15(1), e0227684, 2020. 